\title{
Fault Tolerant Control Using Sliding Modes with On-line Control Allocation *
}

\author{
Halim Alwi, Christopher Edwards \\ Control and Instrumentation Research Group, Department of Engineering, University of Leicester, Leicester LE1 7RH, UK.
}

\begin{abstract}
This paper proposes an on-line sliding mode control allocation scheme for fault tolerant control. The effectiveness level of the actuators is used by the control allocation scheme to redistribute the control signals to the remaining actuators when a fault or failure occurs. The paper provides an analysis of the sliding mode control allocation scheme and determines the nonlinear gain required to maintain sliding. The on-line sliding mode control allocation scheme shows that faults and even certain total actuator failures can be handled directly without reconfiguring the controller. The simulation results show good performance when tested on different fault and failure scenarios. (c)2007 Elsevier Science Ltd. All rights reserved.
\end{abstract}

Key words: fault tolerant control; control allocation; reconfigurable control; sliding modes.

\section{Introduction}

In most safety critical systems e.g. passenger aircraft [4] and modern fighter aircraft [12], there is actuator redundancy. This allows freedom to design fault tolerant control (FTC) systems to maintain stability and acceptable performance during faults and failures. Control allocation $(\mathrm{CA})$ is one approach to manage the actuator redundancy for different control strategies handling actuator faults (see for example [5,8]). There is extensive literature on CA which discusses different algorithms, approaches and applications: [11] discusses two (broadly) linked approaches (linear and quadratic programming) for CA based on finding the 'best solution' to a system of linear equations. The work in [13] compares control allocation with optimal control design for distributing the control effort among redundant actuators. In [6] the authors demonstrate that feedback control systems with redundant actuators can be reduced to a feedback control system without redundancy using a special case of CA known as 'daisy chaining'. In this approach, a subset of the actuators, regarded as the primary actuators are used first, then secondary actuators are used if the primary actuators reach saturation. Other CA approaches

\footnotetext{
‡ This paper was not presented at any IFAC meeting. Corresponding author C. Edwards, Tel.: +44-116-223-1303; fax: +44-116- 252-2619.

Email addresses: ha18@le.ac.uk (Halim Alwi), chris.edwards@le.ac.uk. (Christopher Edwards).

1 This work is supported by an EPSRC grant GR/S70876.
}

taking into account actuator limits are discussed in $[2,3]$.

The work in $[5,8]$ uses CA as a means for fault tolerant control (FTC). The benefits of CA is that the controller structure does not have to be reconfigured in the case of faults and it can deal directly with total actuator failures without requiring reconfiguration/accommodation of the controller: the CA scheme automatically redistributes the control signal. This is the facet of CA that will be explored in this paper. The work in $[17,21]$ provides practical examples of the combination of sliding mode control (SMC) and CA for FTC. The work by Shin et al.[16] uses control allocation ideas, but formulates the problem from an adaptive controller point of view. However neither of these papers provide a detailed stability analysis and discuss sliding mode controller design issues when using control allocation. Recent work by Corradini et al.[7] shows that total failures can be dealt with by SMC schemes provided that there is enough redundancy in the system. However [7] considers exact duplication of actuators to achieve redundancy, whereas in many over actuated systems, the redundant actuators do not have identical dynamics to the 'primary' actuators.

In this paper, a combination of SMC and CA will be explored to achieve FTC. A rigorous design procedure is developed from a theoretical perspective. The control strategy uses the effectiveness level of the actuators, and redistributes the control to the remaining actuators when faults/failures occur. This is the novelty of this paper compared to the work in $[7,17,21]$. 


\section{Controller Design}

\subsection{Problem Formulation}

This paper considers a situation where a fault associated with the actuators develops in a system. It will be assumed that the system subject to actuator faults or failures, can be written as

$\dot{x}(t)=A x(t)+B u(t)-B K(t) u(t)$

where $A \in \mathbb{R}^{n \times n}$ and $B \in \mathbb{R}^{n \times m}$. The effectiveness gain $K(t)=\operatorname{diag}\left(k_{1}(t), \ldots, k_{m}(t)\right)$ where the $k_{i}(t)$ are scalars satisfying $0 \leq k_{i}(t) \leq 1$. These scalars model a decrease in effectiveness of a particular actuator. If $k_{i}(t)=0$, the $i$ th actuator is working perfectly whereas if $k_{i}(t)>0$, a fault is present, and if $k_{i}(t)=1$ the actuator has failed completely. In this paper, information about $K(t)$ will be incorporated into the allocation algorithm . In most CA strategies, the control signal is distributed equally among all the actuators [16] or distributed based on the limits (position and rate) of the actuators [8].

In the literature, the assumption that $\operatorname{Rank}(B)=l<$ $m$ is often employed so that $B$ can be factorized into $B=B_{\nu} N$ where $N \in \mathbb{R}^{m \times l}$. For many systems the assumption, is not valid. However, usually the system states can always be reordered, and the matrix $B$ from (1) can be partitioned as:

$B=\left[\begin{array}{l}B_{1} \\ B_{2}\end{array}\right]$

where $B_{1} \in \mathbb{R}^{(n-l) \times m}$ and $B_{2} \in \mathbb{R}^{l \times m}$ has rank $l$. The partition is in keeping with the notion of splitting the control law from the control allocation task $[13,8]$. Here it is assumed that the matrix $B_{2}$ represents the dominant contribution of the control action on the system, while $B_{1}$ generally will have elements of small magnitude compared with $\left\|B_{2}\right\|$. Compared to the work in [16] where it is assumed that $B_{1}=0$, here $B_{1} \neq 0$ will be considered explicitly in the controller design and in the stability analysis. It will be assumed without loss of generality that the states of the system in (1) have been transformed so that $B_{2} B_{2}^{\mathrm{T}}=I_{l}$ and therefore $\left\|B_{2}\right\|=1$. This is always possible since $\operatorname{rank}\left(B_{2}\right)=l$ by construction. As in [1], let the 'virtual control' $\nu(t)$ be defined as

$\nu(t):=B_{2} u(t)$

so that

$u(t)=B_{2}^{\dagger} \nu(t)$

where the pseudo inverse is chosen as

$B_{2}^{\dagger}:=W B_{2}^{\mathrm{T}}\left(B_{2} W B_{2}^{\mathrm{T}}\right)^{-1}$ and $W \in \mathbb{R}^{m \times m}$ is a symmetric positive definite (s.p.d) diagonal weighting matrix. It can be shown the pseudoinverse in (5) arises from the optimization problem

$\min _{u(t)} u(t)^{\mathrm{T}} W^{-1} u(t) \quad$ subject to $B_{2} u(t)=\nu(t)$

In this paper a novel choice of weighting matrix $W$ will be considered. Specifically, $W$ has been chosen as

$W:=I-K$

and so $W=\operatorname{diag}\left\{w_{1}, \ldots, w_{m}\right\}$ where $w_{i}=1-k_{i}$. Note in a fault free situation $W=I$. As $k_{i} \rightarrow 1, w_{i} \rightarrow 0$ and so the associated component $u_{i}$ in (6) is weighted heavily since $\frac{1}{w_{i}}$ becomes large.

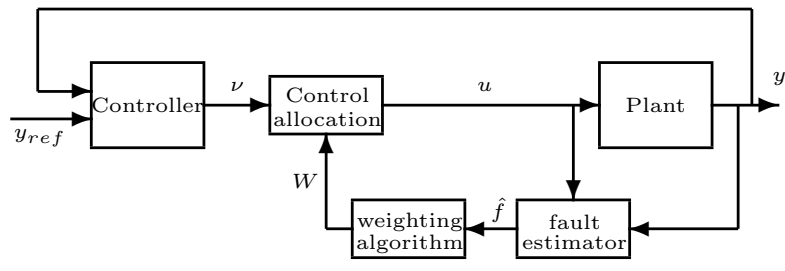

Fig. 1. Control allocation strategy

Figure 1 illustrates the FTC control allocation strategy. The control allocation will depend on the effectiveness of the actuators. The information necessary to compute $W$ on-line can be supplied by a fault reconstruction scheme as described in $[10,19]$ for example, or by using a measurement of the actual actuator deflection compared to the demand which is available in many systems e.g. passenger aircraft [4]. Alternatively other fault reconstruction schemes based on Kalman filters [22] can be used. From (7) if an actuator fault occurs, the weighting $W$ will be changed on-line and the control input $u(t)$ is reallocated to minimize the use of the faulty control surface. In the event of total failure of the $i$ th control surface, $k_{i} \rightarrow 1$ and therefore the $i$ th component of $W^{-1}$ becomes large. Hence, $u_{i}(t)$ is totally re-routed to the other actuators (provided there is enough redundancy in the system).

In this paper, sliding mode control (SMC) techniques [9], have been used to synthesize the 'virtual control' $\nu(t)$. Define a switching function $s(t): \mathbb{R}^{n} \rightarrow \mathbb{R}^{l}$ to be

$$
s(t)=S x(t)
$$

where $S \in \mathbb{R}^{l \times n}$ and $\operatorname{det}\left(S B_{\nu}\right) \neq 0$. Let $\mathcal{S}$ be the hyperplane defined by $\mathcal{S}=\left\{x(t) \in \mathbb{R}^{n}: S x(t)=0\right\}$. If a control law can be developed which forces the closed-loop trajectories onto the surface $\mathcal{S}$ in finite time and constrains the states to remain there, then an ideal sliding motion is said to have been attained [9]. First define

$\hat{\nu}(t):=\left(B_{2} W^{2} B_{2}^{\mathrm{T}}\right)\left(B_{2} W B_{2}^{\mathrm{T}}\right)^{-1} \nu(t)$ 
then as argued in [1], after an appropriate coordinate transformation, $x \mapsto T_{r} x=\hat{x}$, equation (1) becomes:

$$
\left[\begin{array}{l}
\dot{\hat{x}}_{1}(t) \\
\dot{\hat{x}}_{2}(t)
\end{array}\right]=\left[\begin{array}{cc}
\hat{A}_{11} & \hat{A}_{12} \\
\hat{A}_{21} & \hat{A}_{22}
\end{array}\right]\left[\begin{array}{l}
\hat{x}_{1}(t) \\
\hat{x}_{2}(t)
\end{array}\right]+\left[\begin{array}{l}
0 \\
I
\end{array}\right] \hat{\nu}(t)+\left[\begin{array}{c}
B_{1} B_{2}^{N} B_{2}^{+} \\
0
\end{array}\right] \hat{\nu}(t)
$$

where

$B_{2}^{+}:=W^{2} B_{2}^{\mathrm{T}}\left(B_{2} W^{2} B_{2}^{\mathrm{T}}\right)^{-1}$

and

$B_{2}^{N}:=\left(I-B_{2}^{\mathrm{T}} B_{2}\right)$

Proposition 1 There exists a scalar $\gamma_{0}$ which is finite and independent of $W$ such that

$\left\|B_{2}^{+}\right\|=\left\|W^{2} B_{2}^{T}\left(B_{2} W^{2} B_{2}^{T}\right)^{-1}\right\|<\gamma_{0}$

for all $W=\operatorname{diag}\left(w_{1} \ldots w_{m}\right)$ such that $0<w_{i} \leq 1$.

Proof: This follows from a modification of the proof of Theorem 1 in [18]. The work in [18] considers left pseudoinverses but since $\left(B_{2}^{+}\right)^{\mathrm{T}}=\left(B_{2}^{\mathrm{T}}\right)^{+}$, the result follows.

Remark 1: As shown in [18], if $W$ is not diagonal, $\left\|B_{2}^{+}\right\|$ is no longer necessarily bounded.

The virtual control law $\hat{\nu}$ will now be designed based on the nominal fault-free system in which the top partition of the last term in (9) is zero since $\left.B_{1} B_{2}^{N} B_{2}^{+}\right|_{W=I}=0$. In the $\hat{x}(t)$ coordinates in $(9)$, a suitable choice for the sliding surface is

$\hat{S}=S T_{r}^{-1}=\left[M I_{l}\right]$

where $M \in \mathbb{R}^{l \times(n-l)}$ is the design freedom. Introduce another transformation so that $\left(\hat{x}_{1}, \hat{x}_{2}\right) \mapsto\left(\hat{x}_{1}, s(t)\right)$, then equation (9) becomes

$$
\left[\begin{array}{c}
\dot{\hat{x}}_{1}(t) \\
\dot{s}(t)
\end{array}\right]=\left[\begin{array}{cc}
\tilde{A}_{11} & \tilde{A}_{12} \\
\tilde{A}_{21} & \tilde{A}_{22}
\end{array}\right]\left[\begin{array}{c}
\hat{x}_{1}(t) \\
s(t)
\end{array}\right]+\left[\begin{array}{c}
B_{1} B_{2}^{N} B_{2}^{+} \\
I+M B_{1} B_{2}^{N} B_{2}^{+}
\end{array}\right] \hat{\nu}(t)
$$

where $B_{2}^{+}$is defined in (10) and $\tilde{A}_{11}:=\hat{A}_{11}-\hat{A}_{12} M$, $\tilde{A}_{21}:=M \tilde{A}_{11}+\hat{A}_{21}-\hat{A}_{22} M$. If a control law can be designed to induce sliding, then during sliding $\dot{s}(t)=$ $s(t)=0$ and so the equivalent control $[9,20]$ necessary to maintain sliding is obtained from solving for $\hat{\nu}_{e q}(t)$ from the lower equations of (14) to give

$\hat{\nu}_{e q}(t)=-\left(I+M B_{1} B_{2}^{N} B_{2}^{+}\right)^{-1} \tilde{A}_{21} \hat{x}_{1}(t)$

where $B_{2}^{N}$ is defined in (11). Define

$\gamma_{1}:=\left\|M B_{1} B_{2}^{N}\right\|$
It follows $\left\|M B_{1} B_{2}^{N} B_{2}^{+}\right\|<\left\|M B_{1} B_{2}^{N}\right\|\left\|B_{2}^{+}\right\|<\gamma_{0} \gamma_{1}$. Since $B_{2}^{+}$is independent of $M$, the term $\gamma_{0}$ can be calculated a-priori using the boundedness result from Proposition 1 . If $\left(A, B_{\nu}\right)$ is controllable, then $\left(\hat{A}_{11}, \hat{A}_{12}\right)$ is controllable [9] and so $M$ can always be chosen to make $\hat{A}_{11}-\hat{A}_{12} M$ stable. If the design matrix $M$ can also be chosen so that $\gamma_{1}$ from (16) satisfies $\gamma_{0} \gamma_{1}<1$, this guarantees the inverse in (15) exists for all $W$. Substituting (15) into the top partition of (14), yields the following reduced order system which governs the sliding motion:

$\dot{\hat{x}}_{1}(t)=\tilde{A}_{11} \hat{x}_{1}(t)-B_{1} B_{2}^{N} B_{2}^{+}\left(I+M B_{1} B_{2}^{N} B_{2}^{+}\right)^{-1} \tilde{A}_{21} \hat{x}_{1}(t)$

Remark 2: In a fault free condition, $W=I$ and therefore $\left.B_{2}^{+}\right|_{W=I}=B_{2}^{\mathrm{T}}$ since $B_{2} B_{2}^{\mathrm{T}}=I$. Also

$$
B_{2}^{N} B_{2}^{+}=\left(I-B_{2}^{\mathrm{T}} B_{2}\right) B_{2}^{+}=\left(I-B_{2}^{\mathrm{T}} B_{2}\right) B_{2}^{\mathrm{T}}=0
$$

and the system in (17) 'collapses' to $\dot{\hat{x}}_{1}(t)=\tilde{A}_{11} \hat{x}_{1}(t)$ which is the nominal sliding mode reduced order system for which $M$ has been designed to guarantee stability [9].

\subsection{Stability analysis}

The stability of the sliding mode is dependent on the reduced order system (17). Typically in sliding mode control [9] the stability of the system only depends on $\tilde{A}_{11}$ which is guaranteed to be stable by choice of $M$. Note that $M$ can be designed using standard sliding hyperplane design methods (see for example [9]) assuming a nominal no fault condition - i.e. $W=I$ in (9). To facilitate the subsequent analysis, define

$\tilde{G}(\mathrm{~s}):=\tilde{A}_{21}\left(s I-\tilde{A}_{11}\right)^{-1} B_{1} B_{2}^{N}$

where s represents the Laplace variable. By construction the transfer function matrix $\tilde{G}(\mathrm{~s})$ is stable and define

$\gamma_{2}=\|\tilde{G}(\mathrm{~s})\|_{\infty}$

Proposition 2 During a fault or failure condition, for any combination of $0<w_{i} \leq 1$, the closed-loop system will be stable if

$0<\frac{\gamma_{2} \gamma_{0}}{1-\gamma_{1} \gamma_{0}}<1$

where the positive scalar $\gamma_{0}$ is defined in Proposition 1, the positive scalar $\gamma_{1}$ is defined in (16).

Proof: Consider the reduced order system from equation (17) rewritten as follows:

$$
\begin{aligned}
\dot{\hat{x}}_{1}(t) & =\tilde{A}_{11} \hat{x}_{1}(t)-B_{1} B_{2}^{N} \tilde{u}(t) \\
\tilde{y}(t) & =\tilde{A}_{21} \hat{x}_{1}(t)
\end{aligned}
$$


where

$$
\tilde{u}(t):=B_{2}^{+}\left(I+M B_{1} B_{2}^{N} B_{2}^{+}\right)^{-1} \tilde{y}(t)
$$

Let $\tilde{G}(\mathrm{~s})$ be defined as (18). Consequently (17) may be viewed as the closed-loop dynamics of the negative feedback interconnection of $\tilde{G}(\mathrm{~s})$ and the varying (with respect to $W$ ) 'feedback gain' associated with (23). Since (20) is assumed to hold, $\gamma_{0} \gamma_{1}<1$ and it follows that $\left\|M B_{1} B_{2}^{N} B_{2}^{+}\right\|<\left\|M B_{1} B_{2}^{N}\right\|\left\|B_{2}^{+}\right\|<\gamma_{0} \gamma_{1}<1$. Consequently $^{2}, \operatorname{det}\left(I+M B_{1} B_{2}^{N} B_{2}^{+}\right) \neq 0$. Furthermore, using the fact that in general $\left\|(I+X)^{-1}\right\| \leq(I-\|X\|)^{-1}$ if $\|X\|<1$ (page $301[14]$ ), then

$$
\begin{aligned}
\left\|B_{2}^{+}\left(I+M B_{1} B_{2}^{N} B_{2}^{+}\right)^{-1}\right\| & <\left\|B_{2}^{+}\right\|\left\|\left(I+M B_{1} B_{2}^{N} B_{2}^{+}\right)^{-1}\right\| \\
& <\frac{\gamma_{0}}{1-\gamma_{1} \gamma_{0}}
\end{aligned}
$$

From the Small Gain Theorem [15], if

$\|\tilde{G}(\mathrm{~s})\|_{\infty}\left\|B_{2}^{+}\left(I+M B_{1} B_{2}^{N} B_{2}^{+}\right)^{-1}\right\|<1$

then (17) is stable. Using (24) and the fact that $\| \tilde{G}\left(\right.$ s) $\|_{\infty}<\gamma_{2}$, inequality (20) implies (25) holds and so (17) is stable.

Remark 3: Both $\gamma_{1}$ and $\gamma_{2}$ depend on the design of the sliding surface since they depend on $M$, however they are independent of $W$. The scalar $\gamma_{0}$ depends on $W$ but is independent of $M$.

Remark 4: If $B_{1}=0$ (which is an assumption in many schemes: for example [16]), then $\gamma_{1}=0$ and $\gamma_{2}=0$ and Proposition 2 is trivially satisfied. Furthermore, as $\left\|B_{1}\right\| \rightarrow 0$, the scalar $\frac{\gamma_{2} \gamma_{0}}{1-\gamma_{1} \gamma_{0}} \rightarrow 0$ and so the requirements of Proposition 2 are satisfied. This means, for weakly coupled systems in which $\left\|B_{1}\right\|$ is small, the approach will be feasible. The situation where $B_{1}=0$ can be regarded as the special extreme case as $\left\|B_{1}\right\| \rightarrow 0$.

Equation (20) represents a test to guarantee the stability of the closed-loop system when faults occur (i.e. when the $w_{i}$ vary). One important feature is that in order for (17) to hold, the norm of the pseudo-inverse $B_{2}^{+}$which depends on $W$ must be bounded for all $0<w_{i} \leq 1$ (which was proved in Proposition 1).

\footnotetext{
2 Suppose $\operatorname{det}(I+X)=0$, then $\lambda=-1$ is an eigenvalue of $X$ so there exists a $v \in \mathbb{R}^{m}$ s.t. $X v=-v$. Therefore
}

$$
v^{\mathrm{T}} X^{\mathrm{T}} X v=v^{\mathrm{T}} v \Rightarrow \sup _{\|v\|} \frac{v^{\mathrm{T}} X^{\mathrm{T}} X v}{\|v\|^{2}} \geq 1 \Rightarrow\|X\| \geq 1
$$

which is a contradiction since by assumption $\|X\|<1$.

\subsection{Sliding Mode Control laws}

Next, a sliding mode controller is designed based on the system in (14) with respect to $\hat{\nu}$. The proposed control law has a structure given by $\hat{\nu}(t)=\hat{\nu}_{l}(t)+\hat{\nu}_{n}(t)$ where

$\hat{\nu}_{l}(t):=-\tilde{A}_{21} \hat{x}_{1}(t)-\tilde{A}_{22} s(t)$

and the nonlinear component is defined to be

$\hat{\nu}_{n}(t):=-\rho(t, x) \frac{s(t)}{\|s(t)\|} \quad$ for $s(t) \neq 0$

where $s(t)=\hat{S} \hat{x}(t)$.

Proposition 3 Suppose the hyperplane matrix $M$ has been chosen so that $\tilde{A}_{11}=\hat{A}_{11}-\hat{A}_{12} M$ is stable and condition (20) from Proposition 2 holds, then choosing

$\rho(t, x):=\frac{\gamma_{1} \gamma_{0}\left\|\hat{\nu}_{l}(t)\right\|+\eta}{1-\gamma_{1} \gamma_{0}}$

ensures a sliding motion takes place on $\mathcal{S}$ in finite time.

Proof: Notice if $(20)$ holds, then $\gamma_{0} \gamma_{1}<1$ and so the gain defined in (28) is well defined. From (14),

$\dot{s}(t)=\left(I+M B_{1} B_{2}^{N} B_{2}^{+}\right) \hat{\nu}_{n}(t)+\left(M B_{1} B_{2}^{N} B_{2}^{+}\right) \hat{\nu}_{l}(t)$

and so

$$
\begin{aligned}
s^{\mathrm{T}} \dot{s} & =-\rho\|s\|+s^{\mathrm{T}} M B_{1} B_{2}^{N} B_{2}^{+} \hat{\nu}_{n}(t)+s^{\mathrm{T}}\left(M B_{1} B_{2}^{N} B_{2}^{+}\right) \hat{\nu}_{l}(t) \\
& \leq\|s\|\left(\rho\left\|M B_{1} B_{2}^{N} B_{2}^{+}\right\|+\left\|M B_{1} B_{2}^{N} B_{2}^{+}\right\|\left\|\hat{\nu}_{l}(t)\right\|-\rho\right) \\
& \leq\|s\|\left(\rho \gamma_{1} \gamma_{0}+\gamma_{1} \gamma_{0}\left\|\hat{\nu}_{l}(t)\right\|-\rho\right)
\end{aligned}
$$

Choosing $\rho($.$) as in (28), and substituting into (29) im-$ plies $s(t)^{\mathrm{T}} \dot{s}(t) \leq-\eta\|s(t)\|$. This differential inequality is the 'reachability condition' $[9,20]$ and implies $s(t)=0$ in finite time and a sliding motion is maintained for all subsequent time.

It follows that the actual control which is sent to the actuators is resolved from the 'virtual control law' $\nu(t)$ (from (26)-(27)), using (4), (5) and (8). Therefore $u(t)$ is defined as

$u(t)=W B_{2}^{\mathrm{T}}\left(B_{2} W^{2} B_{2}^{\mathrm{T}}\right)^{-1} \hat{\nu}(t)$

i.e. the control which is sent to the actuators depends on the effectiveness gains $k_{i}$ (through the matrix $W$ ).

\subsection{The effect of non-perfect fault reconstruction}

Consider a faulty system represented by equation (1). Let $\bar{K}$ correspond to the estimated reduction of the actuator efficiency based on the information provided by the FDI scheme. Define

$\bar{W}=I-\bar{K}$ 
and suppose $\bar{K} \neq K$, where, as described earlier, $K$ represents the actual reduction in actuator efficiency. Suppose

$W=(I-\Delta) \bar{W}$

were $\Delta=\operatorname{diag}\left(\delta_{1}, \ldots, \delta_{m}\right)$ and (the unknown) $\delta_{i}$ are elements which represent the level of imperfection in the fault reconstruction. Since $(I-K)=W$, from (1)

$\dot{x}(t)=A x(t)+B(I-\Delta) \bar{W} u(t)$

Now suppose $u(t)=\bar{B}_{2}^{\dagger} \nu(t)$ where

$\bar{B}_{2}^{\dagger}:=\bar{W} B_{2}^{\mathrm{T}}\left(B_{2} \bar{W} B_{2}^{\mathrm{T}}\right)^{-1}$

This represents the fact that $\bar{W}$ (i.e. the estimate rather than the true value of $W$ ) will be used to compute the controller. Then (33) becomes

$\dot{x}(t)=A x(t)+B(I-\Delta) \bar{W} \bar{B}_{2}^{\dagger} \nu(t)$

Also define $\bar{\nu}(t)=\left(B_{2} \bar{W} B_{2}^{\mathrm{T}}\right)^{-1} \nu(t)$, then it follows from (35) that

$$
\begin{aligned}
\dot{x}(t)=A x(t)+ & {\left[\begin{array}{c}
B_{1} \bar{W}^{2} B_{2}^{\mathrm{T}} \\
B_{2} \bar{W}^{2} B_{2}^{\mathrm{T}}
\end{array}\right] \bar{\nu}(t)-\left[\begin{array}{c}
B_{1} \Delta \bar{W}^{2} B_{2}^{\mathrm{T}} \\
B_{2} \Delta \bar{W}^{2} B_{2}^{\mathrm{T}}
\end{array}\right] \bar{\nu}(t) } \\
=A x(t)+ & {\left[\begin{array}{c}
B_{1} B_{2}^{\mathrm{T}} \\
I
\end{array}\right] \bar{\nu}(t)-\left[\begin{array}{c}
B_{1}\left(I-\bar{W}^{2}\right) B_{2}^{\mathrm{T}} \\
B_{2}\left(I-\bar{W}^{2}\right) B_{2}^{\mathrm{T}}
\end{array}\right] \bar{\nu}(t) } \\
& -\left[\begin{array}{c}
B_{1} \Delta \bar{W}^{2} B_{2}^{\mathrm{T}} \\
B_{2} \Delta \bar{W}^{2} B_{2}^{\mathrm{T}}
\end{array}\right] \bar{\nu}(t)
\end{aligned}
$$

Notice that equation (36) has an additional term dependent on both the faults and the error in fault reconstruction. Again consider a transformation to regular form using the transformation matrix $T_{r}$ defined in [1]. Equation (36) becomes

$$
\begin{aligned}
\dot{\hat{x}}=\hat{A} \hat{x}(t)+ & {\left[\begin{array}{l}
0 \\
I
\end{array}\right] \bar{\nu}(t)-\left[\begin{array}{c}
-B_{1} B_{2}^{N} \bar{W}^{2} B_{2}^{\mathrm{T}} \\
I-B_{2} \bar{W}^{2} B_{2}^{\mathrm{T}}
\end{array}\right] \bar{\nu}(t) } \\
- & {\left[\begin{array}{c}
B_{1} B_{2}^{N} \Delta \bar{W}^{2} B_{2}^{\mathrm{T}} \\
B_{2} \Delta \bar{W}^{2} B_{2}^{\mathrm{T}}
\end{array}\right] \bar{\nu}(t) } \\
=\hat{A} \hat{x}(t)+ & {\left[\begin{array}{c}
0 \\
B_{2} \bar{W}^{2} B_{2}^{\mathrm{T}}
\end{array}\right] \bar{\nu}(t)+\left[\begin{array}{c}
B_{1} B_{2}^{N} \bar{W}^{2} B_{2}^{\mathrm{T}} \\
0
\end{array}\right] \bar{\nu}(t) } \\
- & {\left[\begin{array}{c}
B_{1} B_{2}^{N} \Delta \bar{W}^{2} B_{2}^{\mathrm{T}} \\
B_{2} \Delta \bar{W}^{2} B_{2}^{\mathrm{T}}
\end{array}\right] \bar{\nu}(t) }
\end{aligned}
$$

where $B_{2}^{N}$ is defined in (11). Define $\hat{\nu}(t):=B_{2} \bar{W}^{2} B_{2}^{\mathrm{T}} \bar{\nu}(t)$ and $\bar{B}_{2}^{+}:=\bar{W}^{2} B_{2}^{\mathrm{T}}\left(B_{2} \bar{W}^{2} B_{2}^{\mathrm{T}}\right)^{-1}$ then (37) becomes

$\dot{\hat{x}}=\hat{A} \hat{x}(t)+\left[\begin{array}{l}0 \\ I\end{array}\right] \hat{\nu}(t)+\left[\begin{array}{c}B_{1} B_{2}^{N}(I-\Delta) \bar{B}_{2}^{+} \\ -B_{2} \Delta \bar{B}_{2}^{+}\end{array}\right] \hat{\nu}(t)$

Consider another coordinate transformation so that $\left(\hat{x}_{1}, \hat{x}_{2}\right) \mapsto\left(\hat{x}_{1}, s(t)\right)$, using $T_{s}$ as defined in [1], then the above becomes

$$
\begin{aligned}
{\left[\begin{array}{c}
\dot{\hat{x}}_{1}(t) \\
\dot{s}(t)
\end{array}\right] } & =\left[\begin{array}{cc}
\tilde{A}_{11} & \tilde{A}_{12} \\
\tilde{A}_{21} & \tilde{A}_{22}
\end{array}\right]\left[\begin{array}{c}
\hat{x}_{1}(t) \\
s(t)
\end{array}\right]+\left[\begin{array}{l}
0 \\
I
\end{array}\right] \hat{\nu}(t) \\
& +\left[\begin{array}{c}
B_{1} B_{2}^{N}(I-\Delta) \bar{B}_{2}^{+} \\
M B_{1} B_{2}^{N}(I-\Delta) \bar{B}_{2}^{+}-B_{2} \Delta \bar{B}_{2}^{+}
\end{array}\right] \hat{\nu}(t)
\end{aligned}
$$

where $\tilde{A}_{11}:=\hat{A}_{11}-\hat{A}_{12} M, \tilde{A}_{21}:=M \tilde{A}_{11}+\hat{A}_{21}-\hat{A}_{22}$.

If a control law can be designed to induce sliding, then during sliding $\dot{s}(t)=s(t)=0$ and so the equivalent control $[9,20]$ necessary to maintain sliding is obtained from solving for $\hat{\nu}_{e q}(t)$ from the lower equations of (39) to give

$\hat{\nu}_{e q}(t)=-\Omega^{-1} \tilde{A}_{21} \hat{x}_{1}(t)$

where

$\Omega=\left(I+M B_{1} B_{2}^{N}(I-\Delta) \bar{B}_{2}^{+}-B_{2} \Delta \bar{B}_{2}^{+}\right)$

Substituting into the first equation of (39) gives the following reduced order system [9]:

$\dot{\hat{x}}_{1}(t)=\tilde{A}_{11} \hat{x}_{1}(t)-B_{1} B_{2}^{N}(I-\Delta) \bar{B}_{2}^{+} \Omega^{-1} \tilde{A}_{21} \hat{x}_{1}(t)$

Remark 5: If the information on the actual degradation of the control surface efficiency is 'perfect', then $\Delta=0$, and (42) reduces to (17) in the stability analysis that follows. However in the event of non-perfect fault reconstruction, there is a bound on $\Delta$ for which stability is still guaranteed.

Proposition 4 Assume (as in Proposition 2), that equation (20) holds. During a fault or failure condition, for any combinations of $0<w_{i} \leq 1$, the closed-loop system will be stable if the mismatch between the actual and reconstructed fault $\Delta$ satisfies:

$\|\Delta\|<\frac{1-\gamma_{1} \gamma_{0}-\gamma_{2} \gamma_{0}}{\gamma_{0}\left(\gamma_{1}+\gamma_{2}+1\right)}$

where $\gamma_{0}$ and $\gamma_{1}$ are defined in Proposition 1 and 2 respectively and $\gamma_{2}$ is defined in (19). 
Proof: Consider the reduced order system from equation (42) which can be rewritten as (21)-(23), where now

$\tilde{u}(t):=(I-\Delta) \bar{B}_{2}^{+}\left(I+M B_{1} B_{2}^{N}(I-\Delta) \bar{B}_{2}^{+}-B_{2} \Delta \bar{B}_{2}^{+}\right)^{-1} \tilde{y}(t)(44)$

Assume that (20) and (43) hold. Inequality (43) implies

$\|\Delta\|<\frac{1-\gamma_{1} \gamma_{0}}{\gamma_{0}\left(\gamma_{1}+1\right)}$

because

$\frac{1-\gamma_{1} \gamma_{0}}{\gamma_{0}\left(\gamma_{1}+1\right)}>\frac{1-\gamma_{1} \gamma_{0}-\gamma_{2} \gamma_{0}}{\gamma_{0}\left(\gamma_{1}+\gamma_{2}+1\right)}>0$

Since

$$
\begin{gathered}
\left\|M B_{1} B_{2}^{N}(I-\Delta) \bar{B}_{2}^{+}-B_{2} \Delta \bar{B}_{2}^{+}\right\| \leq \\
+\left\|M B_{1} B_{2}^{N}\right\|(1+\|\Delta\|)\left\|\bar{B}_{2}^{+}\right\| \\
+\left\|B_{2}\right\|\|\Delta\|\left\|\bar{B}_{2}^{+}\right\|
\end{gathered}
$$

and in addition $\left\|B_{2}\right\|=1$ and $\left\|\bar{B}_{2}^{+}\right\| \leq \gamma_{0}$ (since $\left\|B_{2}^{+}\right\| \leq$ $\gamma_{0} \Rightarrow\left\|\bar{B}_{2}^{+}\right\| \leq \gamma_{0}$ ), inequality (45) implies

$\left\|M B_{1} B_{2}^{N}(I-\Delta) \bar{B}_{2}^{+}-B_{2} \Delta \bar{B}_{2}^{+}\right\| \leq \gamma_{1} \gamma_{0}+\|\Delta\| \gamma_{0}\left(\gamma_{1}+1\right)<1(47)$

Therefore $\Omega^{-1}$ exists for all $0<w_{i} \leq 1$. Furthermore, using arguments similar to those in Proposition 2

$\left\|(I-\Delta) \bar{B}_{2}^{+} \Omega^{-1}\right\|<\frac{(1+\|\Delta\|) \gamma_{0}}{\left(1-\gamma_{1}(1+\|\Delta\|) \gamma_{0}-\|\Delta\| \gamma_{0}\right)}$

From Small Gain Theorem, since (42) is the closed-loop system obtained from $\tilde{G}(\mathrm{~s})$ interconnected with (44) if

$\frac{(1+\|\Delta\|) \gamma_{0}}{\left(1-\gamma_{1}(1+\|\Delta\|) \gamma_{0}-\|\Delta\| \gamma_{0}\right)}<\frac{1}{\gamma_{2}}$

holds, then (42) is stable. By direct manipulation, (49) holds if (43) holds, and the proof is complete.

As in $§ 2.3$, a sliding mode controller will now be designed based on the 'virtual' system in (39) with respect to $\hat{\nu}$, as defined in (26) and (27).

Proposition 5 Suppose that the hyperplane matrix $M$ has been chosen so that $\tilde{A}_{11}=\hat{A}_{11}-\hat{A}_{12} M$ is stable and

$\|\Delta\| \leq \Delta_{\max }<\frac{1-\gamma_{1} \gamma_{0}}{\gamma_{0}\left(1+\gamma_{1}\right)}$

where $\gamma_{0}, \gamma_{1}$ and $\gamma_{2}$ are defined in (12), (16) and (19), and $\Delta_{\max }$ is a fixed positive scalar. Then choosing

$\rho(t, x)=\frac{\left(\gamma_{1}\left(1+\Delta_{\max }\right) \gamma_{0}+\Delta_{\max } \gamma_{0}\right)\left\|\hat{\nu}_{l}(t)\right\|+\eta}{1-\gamma_{1}\left(1+\Delta_{\max }\right) \gamma_{0}-\Delta_{\max } \gamma_{0}}$

ensures a sliding motion takes place on $\mathcal{S}$ in finite time.
Proof: The assumption on $\Delta$ in (50) implies $\gamma_{0} \gamma_{1}+(1+$ $\left.\gamma_{1}\right) \Delta_{\max } \gamma_{0}<1$ and so the gain $\rho(t, x)$ in (51) is well defined. From (39),

$$
\begin{aligned}
\dot{s}(t)= & \tilde{A}_{21} \hat{x}_{1}(t)+\tilde{A}_{22} s(t) \\
& +\left(I+M B_{1} B_{2}^{N}(I-\Delta) \bar{B}_{2}^{+}-B_{2} \Delta \bar{B}_{2}^{+}\right) \hat{\nu}(t) \\
= & \left(I+M B_{1} B_{2}^{N}(I-\Delta) \bar{B}_{2}^{+}-B_{2} \Delta \bar{B}_{2}^{+}\right) \hat{\nu}_{n}(t) \\
& +\left(M B_{1} B_{2}^{N}(I-\Delta) \bar{B}_{2}^{+}-B_{2} \Delta \bar{B}_{2}^{+}\right) \hat{\nu}_{l}(t)
\end{aligned}
$$

after substituting for $\nu_{l}(t)$ from (26). Consequently substituting $\nu_{n}(t)$ from (27) into the above yield

$$
\begin{aligned}
s^{\mathrm{T}} \dot{s}= & -\rho\|s\|+s^{\mathrm{T}}\left(M B_{1} B_{2}^{N}(I-\Delta) \bar{B}_{2}^{+}-B_{2} \Delta \bar{B}_{2}^{+}\right) \hat{\nu}_{n}(t) \\
& +s^{\mathrm{T}}\left(M B_{1} B_{2}^{N}(I-\Delta) \bar{B}_{2}^{+}-B_{2} \Delta \bar{B}_{2}^{+}\right) \hat{\nu}_{l}(t) \\
\leq & \|s\|\left(\rho\left\|M B_{1} B_{2}^{N}(I-\Delta) \bar{B}_{2}^{+}-B_{2} \Delta \bar{B}_{2}^{+}\right\|\right. \\
& \left.+\left\|M B_{1} B_{2}^{N}(I-\Delta) \bar{B}_{2}^{+}-B_{2} \Delta \bar{B}_{2}^{+}\right\|\left\|\hat{\nu}_{l}(t)\right\|-\rho\right) \\
\leq & \|s\|\left(-\rho\left(1-\gamma_{1}\left(1+\Delta_{\max }\right) \gamma_{0}-\Delta_{\max } \gamma_{0}\right)\right. \\
& \left.+\left(\gamma_{1}\left(1+\Delta_{\max }\right) \gamma_{0}+\Delta_{\max } \gamma_{0}\right)\left\|\hat{\nu}_{l}(t)\right\|\right)
\end{aligned}
$$

So choosing $\rho($.$) as described in (51) and substituting$ into (52) implies

$s(t)^{\mathrm{T}} \dot{s}(t) \leq-\eta\|s(t)\|$

Again, as in $\S 2.3$, the differential inequality (53) implies that the 'reachability condition' is achieved. Therefore $s(t)=0$ in finite time and a sliding motion is maintained for all subsequent time.

\section{An Example}

The ADMIRE model has been used by several researchers (e.g. [13]) and within the Group for Aeronautical Research and Technology in Europe (GARTEUR) AG11 and AG12 [12]. The linear model used here has been obtained at a low speed flight condition of Mach 0.22 at an altitude of $3000 \mathrm{~m}$ and is similar to the one in [13]. The states are $x=\left[\begin{array}{lll}\alpha & \beta & p\end{array} \quad r\right]^{\mathrm{T}}$ with controlled outputs $\alpha, \beta, p$; where $\alpha$ is angle of attack (AoA) (rad), $\beta$ is sideslip angle ( $\mathrm{rad}), p$ is roll rate $(\mathrm{rad} / \mathrm{sec}), q$ is pitch rate $(\mathrm{rad} / \mathrm{sec})$ and $r$ is yaw rate $(\mathrm{rad} / \mathrm{sec})$. The control surfaces are $\delta=\left[\begin{array}{llll}\delta_{c} & \delta_{r e} & \delta_{l e} & \delta_{r}\end{array}\right]^{\mathrm{T}}$, which represent the deflections ( $\mathrm{rad}$ ) of the canard, right elevon, left elevon and rudder respectively. A linearized model [13] is: 
0.0819. Therefore from Proposition 2,

$$
\begin{aligned}
A & =\left[\begin{array}{rrrrr}
-0.5432 & 0.0137 & 0 & 0.9778 & 0 \\
0 & -0.1179 & 0.2215 & 0 & -0.9661 \\
0 & -10.5128 & -0.9967 & 0 & 0.6176 \\
2.6221 & -0.0030 & 0 & -0.5057 & 0 \\
0 & 0.7075 & -0.0939 & 0 & -0.2127
\end{array}\right] \\
B & \left.=\left[\begin{array}{rrrr}
0.0069 & -0.0866 & -0.0866 & 0.0004 \\
0 & 0.0119 & -0.0119 & 0.0287 \\
0 & -4.2423 & 4.2423 & 1.4871 \\
1.6532 & -1.2735 & -1.2735 & 0.0024 \\
0 & -0.2805 & 0.2805 & -0.8823
\end{array}\right]\right\} B_{1}
\end{aligned}
$$

The partition of $B$ in (55) shows the terms $B_{1}$ and $B_{2}$ (although a further change of coordinate is necessary to scale $B_{2}$ to ensure $\left.B_{2} B_{2}^{\mathrm{T}}=I\right)$. It can be shown that in the coordinates in which $\left\|B_{2}\right\|=1,\left\|B_{1}\right\|=0.1227$ and so the dominant effect of the control signal is through the $B_{2}$ channels. To include a tracking facility, integral action as described in $[1,9]$ has been included. A quadratic optimal design has been used to obtain the sliding surface matrix $S_{a}$ (see for example [20,9]). The symmetric positive definite weighting matrix has been chosen as $Q=\operatorname{diag}(20,20,20,7,10,10,1,1)$. In the simulations the discontinuity in the nonlinear control term has been smoothed by using a sigmoidal approximation where the smoothing scalar has been chosen as 0.001 (see for example $\S 3.7$ in [9]). This removes the discontinuity and introduces a further degree of tuning to accommodate the actuator limits, especially during actuator fault or failure conditions.

In normal flight, either the canard or elevons (left \& right) are sufficient to provide the pitch moment and therefore redundancy is available. In the event of faults or failures, elevons can replace the canard to obtain a pitch moment. However for roll, the elevons will become the only active control surface (the rudder is used for yaw). During the design stage, and based on analysis from $(28)$, it was found that, $\operatorname{rank}\left(B_{2} W B_{2}^{\mathrm{T}}\right)<3$ when the rudder completely fails or any two surfaces from the set consisting of the canard and the left and right elevons completely fail. This is an expected result since there is no redundancy for the rudder to provide yaw; and when two actuators fail from either the canard or elevons, it means that there is no redundancy left in the system and all possible actuators to provide pitch or roll have failed. Based on this assumption, it can be verified from a numerical search that $\gamma_{0}=2.0913$. Simple calculations show that $\gamma_{1}=0.0980$, therefore $\gamma_{1} \gamma_{0}=0.2050<1$ and so, the requirement of Proposition 2 is satisfied. Also for this particular choice of sliding surface $\|\tilde{G}(\mathrm{~s})\|_{\infty}<\gamma_{2}=$

$$
\frac{\gamma_{2} \gamma_{0}}{1-\gamma_{1} \gamma_{0}}=0.2154<1
$$

which shows the closed-loop system is stable for all $0<w_{i} \leq 1$. From Proposition 4, the limits of the tolerable mismatch between the actual and estimated fault signal (for guaranteed stability) is $\Delta_{\max }=0.3519$. Here it is assumed that direct measurements of the actuator deflections are not available and a sliding mode reconstruction approach as described in [1] has been used.

In the following simulations (which assume that there is no saturation or rate limits on the actuators), the linear aircraft model from [13] undertakes a manoeuvre called ' $\alpha$ roll' [13], where a step demand of magnitude $10 \mathrm{deg}$ is applied to $\alpha$ during 1-5 sec and a step of $150 \mathrm{deg}$ for $p$ is applied during 3-7 sec. (There is no reference command for $\beta$ - see Figure 2(a)). Figures 2(a), 2(b), 2(c) and $2(\mathrm{~d})$ show the responses of the closed-loop system under 11 different canard fault conditions ranging from $0 \% \rightarrow 100 \%$ (including total failure). It can be seen that the control signal is systematically re-routed to the right and left elevon (Figure 2(b)). The tracking responses (Figure 2(a)) show no degradation in performance. Figure 2(a) shows that the observer designed for fault reconstruction, tracks the plant output 'perfectly'. Figure 2 (c) shows the evolution of the fault reconstruction signal from the observer. These signals are used for the online control allocation through the term $W$ as shown in Figure 2(d).

\section{Conclusions}

This paper has presented an on-line sliding mode control allocation scheme for fault tolerant control. The effectiveness level of the actuators is used by the control allocation scheme to redistribute the control signals to the remaining actuators when a fault or failure occurs. This paper has provided an analysis of the proposed sliding mode control allocation scheme and has determined the nonlinear gain required to maintain sliding. The on-line sliding mode control allocation scheme implemented on the ADMIRE model has shown that fault and total actuator failures can be handled directly without reconfiguring the controller.

\section{References}

[1] H. Alwi and C. Edwards. Fault tolerant control using sliding mode with on-line control allocation. In Proc. Conf. Decision Contr., 2006.

[2] K.A. Bordignon and W.C. Durham. Closed-form solutions to constrained control allocation problem. J. Guidance, Contr., Dyn., 18:1000-7, 1995. 
[3] J.D. Boskovic and R.K. Mehra. Control allocation in overactuated aircraft under position and rate limiting. In Proc. Amer. Contr. Conf., pages 791-796, 2002.

[4] D. Brière and P. Traverse. Airbus A320/A330/A340 electrical flight controls: A family of fault-tolerant systems. Digest of Papers FTCS-23 The 23rd International Symposium on Fault-Tolerant Computing, pages 616-623, 1993.

[5] J. Buffington, P. Chandler, and M. Pachter. On-line system identification for aircraft with distributed control effectors. Int. J. Robust Nonlinear Control, 9:1033-1049, 1999.

[6] J.M. Buffington and D.F. Enns. Lyapunov stability analysis of daisy chain control allocation. J. Guidance, Contr., Dyn., 19:1226-30, 1996.

[7] M.L. Corradini, G. Orlando, and G. Parlangeli. A fault tolerant sliding mode contoller for accommodating actuator failures. In Proc. Conf. Decision Contr., 2005.

[8] J. Davidson, F. Lallman, and W. Bundick. Real-time adaptive control allocation applied to a high performance aircraft. In 5th SIAM Conf. on Contr. ES Its Application, 2001.

[9] C. Edwards and S.K. Spurgeon. Sliding Mode Control: Theory and Applications. Taylor \& Francis, 1998.

[10] C. Edwards, S.K. Spurgeon, and R.J. Patton. Sliding mode observers for fault detection. Automatica, 36:541-553, 2000.

[11] D. Enns. Control allocation approaches. In Proc. AIAA Guidance, Navigation, Contr. Conf., pages 98-108, 1998.

[12] L. Forssell and U. Nilsson. ADMIRE, the aero-data model in a research environment version 4.0, model description. Technical report, Swedish Defence Agency (FOI), 2005.

[13] O. Härkegård and S. T. Glad. Resolving actuator redundancy - optimal control vs. control allocation. Automatica, 41:137$144,2005$.

[14] R.A. Horn and C.R. Johnson. Matrix Analysis. Cambridge University Press, 1990.

[15] H.K. Khalil. Nonlinear Systems. Prentice Hall, Englewood Cliffs NJ., 1992.

[16] D. Shin, G. Moon, and Y. Kim. Design of reconfigurable flight control system using adaptive sliding mode control: actuator fault. Proc. IMechE, Part G: (J. Aerospace Engineering), 219:321-328, 2005.

[17] Y. Shtessel, J. Buffington, and S. Banda. Tailless aircraft flight control using multiple time scale re-configurable sliding modes. IEEE Trans. Control Syst. Technol., 10:288-296, 2002.

[18] G. W. Stewart. On scaled projections and pseudoinverses. Linear Algebra and Its Applications, 112:189-193, 1989.

[19] C.P. Tan and C. Edwards. Sliding mode observers for robust detection and reconstruction of actuator and sensor faults. Int. J. Robust Nonlinear Control, 13:443-463, 2003.

[20] V.I. Utkin. Sliding Modes in Control Optimization. SpringerVerlag, Berlin, 1992.

[21] S.R. Wells and R.A. Hess. Multi-input/multi-output sliding mode control for a tailless fighter aircraft. J. Guidance, Contr., Dyn., 26:463-473, 2003.

[22] Y. M. Zhang and J. Jiang. Active fault-tolerant control system against partial actuator failures. IEE Proc. Contr. Theory \& Applications, 149:95-104, 2002.
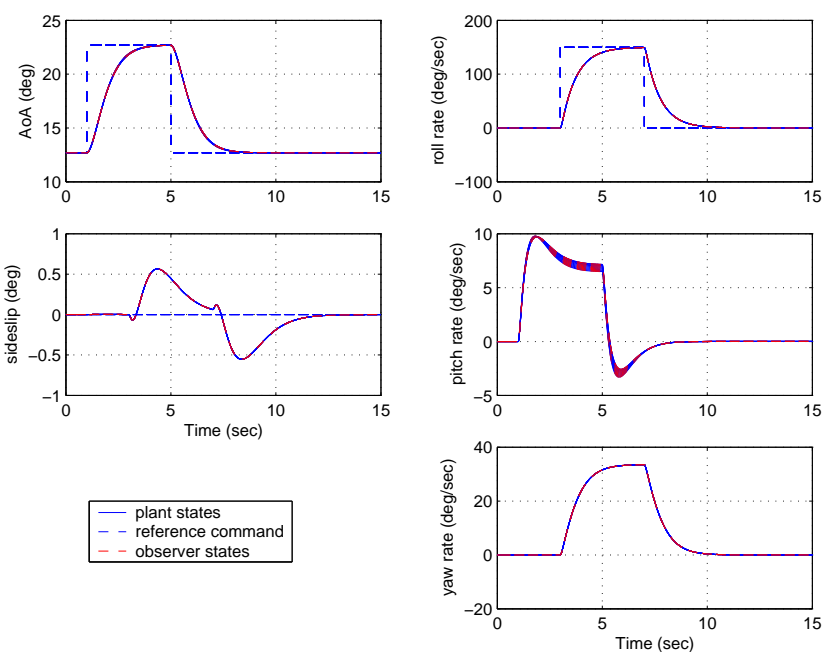

(a) canard fault evolution: states
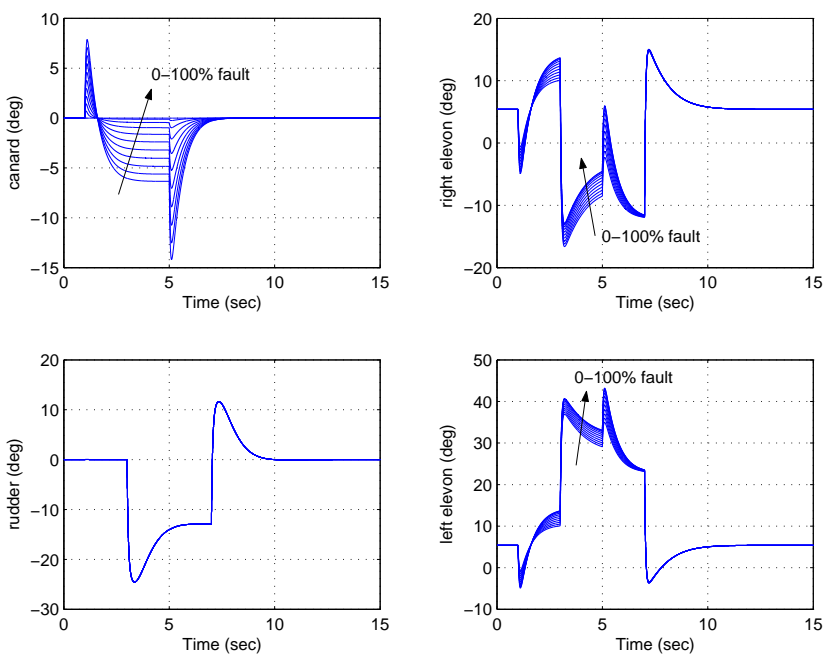

(b) canard fault evolution: actuator deflection

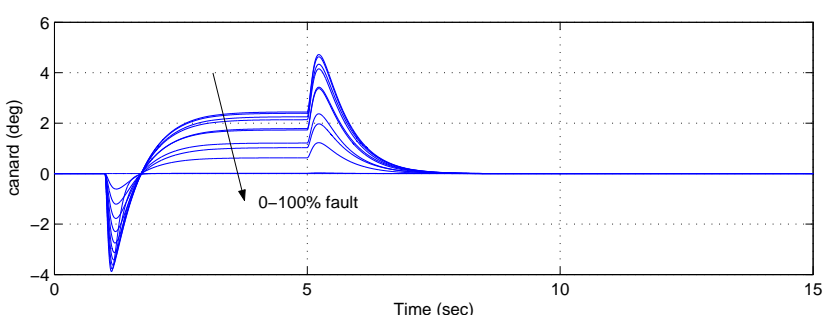

(c) canard fault evolution: fault recons. $\left(k_{i} u_{i}(t)\right)$ signals

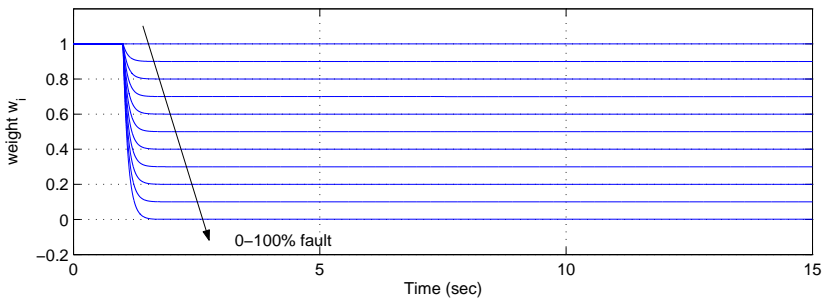

(d) canard fault evolution: weight $w_{i}$ signals

Fig. 2. Responses of fault \& failure on canard 